\title{
Evidence in the Age of Social Media
}

\author{
David B. Nash, MD, MBA
}

$\mathbf{I}^{\mathrm{s}}$ N OUR INCREASINGLY "WIRED" society, many of us are less inclined to go to the source for evidence. Rather, we gravitate to the tweets, blogs, and talk shows that are most likely to support our world view and validate our preconceived notions. This is especially true when the topic is an emotionally charged one without $100 \%$ conclusive evidence.

Reviewing the material for this supplement, I was reminded of "Story as Evidence, Evidence as Story,"1 an excellent article by Louise Aronson, MD, MFA, illustrating how even the most compelling evidence can be misconstrued-and even rejected-on the basis of how it is "packaged" for the media.

Aronson's article recounted a radio news program with a clinician-scientist guest discussing recent articles that challenged "our current beliefs about and uses of mammography, including underreporting of harms, high rates of false-positives, screening-age controversies, and how women would make different choices if they knew the true risks." Initially, the host and callers clearly respected the guest, who deftly reduced complex data to easily understood information and came across as smart, caring, and articulate.

The dynamic changed when a caller related an emotionally charged story of her 42-year-old sister who was treated successfully for breast cancer and "alive to see her kids grow up-all thanks to the mammogram." Although the guest hadn't taken an anti-mammogram position, the caller had come to that conclusion. After expressing sympathy for the caller and her family, the guest returned to the evidence, explaining its implications for individuals and populations and noting the need for further study concerning the appropriateness of mammograms before the age of 40 .

In the final minutes, the conversation devolved into "a subtly tense discourse," with the host and callers challenging the guest-and the guest defending her position by citing additional evidence. Aronson observes that what the radio show listeners had needed was an alternate anecdote that supported the science-in this case, the story of her close friend whose mammogram led to a spate of noninvasive tests and unnecessary invasive procedures that left her with permanent disfigurement, large medical copays, lost time from work, and ongoing anxiety and stress.

This account struck a chord with me. Like most in the health-care professions and policy arena, I expect and rely heavily on objectivity; big data; and rational, evidencebased concepts in my communications. For me, an "expert" is a respected clinician or researcher with extensive experience on a particular topic. Aronson points out that in the public sector, "a single, well-told story of human suffering trumps the most eloquent explanation of a large-scale trial." Her message is a clear signal to the medical communityespecially those of us in academic settings. In addition to reporting the evidence to our peers in journals and at scientific meetings, we must learn how to communicate the evidence persuasively to broader audiences across multiple settings and media.

Policy decisions involving population health are among the most difficult, especially when there is conflicting evidence and when the topic is emotionally charged. It became abundantly clear to me that this Journal has a pivotal role to play in evaluating the science and communicating the evidence that will inform those decisions. To that end, this supplement contains a well-researched, balanced discussion of the evidence on a controversial topic-overdiagnosis as it relates to mammography screening practices_-and presents a strong case for considering newer screening technologies as important tools for reducing the likelihood of overdiagnosis of breast cancer. It also helps lay the groundwork for important conversations regarding value; for example, flexible insurance designs that enable clinicians to recommend appropriate tests based on individual patient factors and long-term outcomes rather than short-term costs.

I am sure that our readers will find the supplement enlightening, and I commend the authors on their fine work.

\section{Reference}

1. Aronson L. Story as evidence, evidence as story. JAMA 2015;314:125-126. 